\title{
Development of a predictive nomogram for early recurrence of hepatocellular carcinoma in patients undergoing liver transplantation
}

\author{
Ensi Ma ${ }^{1,2 \#}$, Jianhua $\mathrm{Li}^{1,2 \#}$, Hao Xing ${ }^{1,2 \#}$, Ruidong $\mathrm{Li}^{1,2}$, Conghuan Shen ${ }^{1,2}$, Quanbao Zhang ${ }^{1,2}$, Zhenyu Ma ${ }^{1,2}$, \\ Yifeng Tao ${ }^{1,2}$, Lunxiu Qin ${ }^{1}$, Jing Zhao ${ }^{1}$, Zhengxin Wang ${ }^{1,2}$ \\ ${ }^{1}$ Department of General Surgery, Huashan Hospital, Fudan University, Shanghai, China; ${ }^{2}$ Institute of Organ Transplantation, Fudan University, \\ Shanghai, China \\ Contributions: (I) Conception and design: E Ma, J Li, H Xing; (II) Administrative support: Z Wang, L Qin, J Zhao; (III) Provision of study materials \\ or patients: R Li, C Shen, Q Zhang, Z Ma, Y Tao; (IV) Collection and assembly of data: E Ma, J Li, H Xing, R Li, C Shen, Q Zhang, Z Ma, Y Tao; (V) \\ Data analysis and interpretation: E Ma, J Li, H Xing; (VI) Manuscript writing: All authors; (VII) Final approval of manuscript: All authors. \\ \#These authors contributed equally to this work. \\ Correspondence to: Jing Zhao; Zhengxin Wang. Department of General Surgery, Huashan Hospital, Fudan University, No. 12 Middle Urumqi Road, \\ Shanghai 200040, China. Email: JingZhao@fudan.edu.cn; wangzhengxin@huashan.org.cn.
}

Background: An individual prognostic model that includes inflammation caused by the delayed recovery of liver function after surgery for the early recurrence of hepatocellular carcinoma (HCC) following liver transplantation (LT) has not been well determined. Our aim was to develop a nomogram model for predicting individual survival and early recurrence following LT for patients.

Methods: Retrospective data, including clinical pathology and follow-up data, on HCC patients were collected between October 2016 and October 2019 at Huashan Hospital Affiliated to Fudan University. A nomogram estimating recurrence post-transplantation was constructed using multivariate Cox regression analysis.

Results: A total of 210 patients were included in the present study. The multivariate estimators of recurrence consisted of age, maximum tumor diameter, tumor thrombus, microvascular invasion (MVI), alanine aminotransferase and alpha-fetoprotein on postoperative day 7 . Nomogram of recurrencefree survival was developed. The calibration and discrimination of the novel model were assessed with the calibration curves and concordance index (C-index). Its reliability and advantages were evaluated by comparing it with the conventional American Joint Committee on Cancer (AJCC) 8th edition staging system using integrated discrimination improvement (IDI) and net reclassification improvement (NRI). In comparison to the AJCC 8th edition staging system, the C-index (development set: 0.796 vs. 0.643 , validation set: 0.741 vs. 0.563 ), the area under the receiver operating characteristic curve (AUC) of the validation set (1-year AUC: 0.732 vs. 0.586, 2-year AUC: 0.705 vs. 0.504), the development set (1-year AUC: 0.799 vs. 0.551, 2-year AUC: 0.801 vs. 0.512), and this model's calibration plots all showed improved performance. In addition, NRI and IDI verified that the nomogram is an accurate prognostic tool. Subsequently, a web calculator was generated to assess the risk of tumor recurrence post-LT.

Conclusions: The nomogram, based on clinical and pathological factors, showed good accuracy in estimating prognostic recurrence and can be used to guide individual patient follow-up and treatment.

Keywords: Liver transplantation (LT); prognosis; hepatocellular carcinoma (HCC)

Submitted Dec 18, 2020. Accepted for publication Mar 03, 2021.

doi: $10.21037 / \mathrm{atm}-21-334$

View this article at: http://dx.doi.org/10.21037/atm-21-334 


\section{Introduction}

Hepatocellular carcinoma (HCC) is the fourth most common cause of cancer-related death and the sixth most prevalent malignant tumor (1). As a solid tumor, HCC has a poor rate of excision and a high recurrence rate (2). As it can cure both primary liver disease and HCC itself, liver transplantation (LT) has gradually become the most efficacious method for treating HCC, especially for patients with liver cirrhosis. In 1996, Mazzaferro et al. presented the widely accepted Milan criteria (3). Based on these criteria, more than $70 \%$ of patients can achieve a excellent survival rate of 5 years (4). To enable more patients to benefit from LT treatment, additional transplantation criteria, such as UCSF (University of California, San Francisco), Up-to-7 (HCC with 7 as the sum of the size of the largest tumor and the number of tumors), and TTV(total tumor volume), have been proposed by transplant centers worldwide (5). Numerous expanded criteria for HCC LT adaptation have enabled some patients with moderate, or even locally progressed HCC, to be cured. A previously published study found that HCC LT patients who met the UCSF, TTV, and Up-to-7 criteria had a 5 -year survival rate of $75 \%$, which was similar to the prognosis of LT patients with HCC who fulfilled the stringent Milan criteria (4).

However, the ever-expanding criteria of HCC LT also increase the risk of metastasis and recurrence after surgery (6). Recurrence after HCC LT is among the most commonly seen causes of mortality in the surgical population (7). The 5-year HCC recurrence rate after LT is $20-57.8 \%$, while the overall median survival time after recurrence is only 12.97 months. Some remarkable progress has recently been made in anti-cancer strategies and drugs, such as antiviral therapy, new targeted drugs, and mTOR (the mammalian target of rapamycin) inhibitors. The efficacy of such treatments is not enough to meet needs; the response rate is not high. Therefore, a precise prediction of a population at high risk of postoperative recurrence will guide the development of an efficient follow-up monitoring program and prevention strategies.

Patients who meet the Milan criteria still have a 15-20\% chance of tumor recurrence after LT. Therefore, it is not enough to predict relapse, metastasis based solely on tumor size and number. As well as HCC LT adaptation criteria, the risk factors related to relapse, metastasis after HCC LT mainly include tumor-related factors, patient-related factors, and perioperative treatment. The tumor level, including tumor diameter, number, envelope, microvascular invasion (MVI), portal venous tumor thrombosis and tumor differentiation, are high-risk factors for tumor recurrence and metastasis. Markers represented by alpha-fetoprotein (AFP), neutrophil lymphocyte ratio, and genomic sequencing have been considered increasingly important in recent years. In particular, dynamic changes in AFP, which have been included in many criteria for HCC indications, have been confirmed by a large number of studies to play a vital part in predicting, checking, and diagnosing recurrence after HCC LT. Patient-level factors mainly include nonalcoholic fatty liver disease, viral hepatitis infection, and obesity. Perioperative period treatment-level factors include the length of preoperative waiting time, whether or not bridging treatment was received, donor age, cold ischemia time, surgical techniques, postoperative immunosuppression and targeted treatment (8). These indicators are commonly seen as clinical-pathological factors in the preoperative and intraoperative period. However, the relationship between postoperative liver function recovery and HCC recurrence is still not clear. The combination of multi-risk factors has the advantage of high predictive effectiveness; for example, the RETREAT (The Risk Estimation of Tumor Recurrence After Transplant) score divides patients into 4 risk levels by including AFP, MVI, tumor max diameter, and tumor number. However, those research included patients with a history of hepatitis $\mathrm{C}$ virus $(\mathrm{HCV})$, and individual predictions with continuous personal risk prediction could not be made as a result of this (9).

Nomogram is a visual chart representation of Cox regression analyses. It has the advantages of high accuracy and visualization, and is easy to populate with various clinical pathology factors and other factors, yielding individual risk predictions. Nomogram is widely used in clinical decision-making. Several researchers have applied nomograms to predict HCC recurrence metastasis following LT, such as UCLA's single-center 865 cases of HCC postLT recurrence metastasis (10). However, the participants included in that study were mainly from western population. Chinese Huaxi Hospital and Renji Hospital have also generated nomograms for patient prognosis after HCC LT $(11,12)$, but patients' postoperative liver function was not included in these studies.

The aim of the present study was to incorporate clinicalpathological factors, including postoperative liver function, to establish and verify a risk predictive nomogram model of recurrence after HCC LT in China for individualized patient recurrence risk assessment.

We present the following article in accordance with the 
TRIPOD reporting checklist (available at http://dx.doi. org/10.21037/atm-21-334).

\section{Methods}

\section{Participants}

From February 2016 until October 2019, 210 patients with HCC who underwent LT at the Huashan Hospital Affiliated to Fudan University, were included in the study. All the patients were followed-up until December 2019 or recurrence. Inclusion criteria consisted of patients with histologically proven HCC without major vascular invasion. The exclusion criteria were HCC combined with other malignant tumors, extrahepatic metastasis, death within 3 months after surgery, and incomplete follow-up information. Of the included patients, two-thirds served as the development set and were randomly selected, while the remaining one-third was the validation set. All donor livers were DCD (donors of cardiac death) matched by the China Organ Transplant Response System. No organs from prisoner under sentence of death were transplanted and reported in this study. All transplantations received approval from the Ethics Committee of Huashan Hospital Affiliated to the Fudan University. The retrospective study was performed according to the Declaration of Helsinki (as revised in 2013) and approval was received from the Ethics Committee of Huashan Hospital Affiliated to Fudan University (No. KY-2019-511). Written consent to publish this information has been obtained from all participants.

\section{Immunosuppression and antiviral protocol post-LT}

The principal scheme of immunosuppressive medication included tacrolimus (TAC), corticosteroids, ciclosporin, mycophenolate mofetil (MMF), and sirolimus. During the first 7 postoperative days, methylprednisolone was given intravenously with a gradually decreasing dosage. Intravenous corticosteroid was switched to prednisone orally when patients were discharged from hospital, and then discontinued 3-6 months following LT. The first dosage of TAC was $0.05-0.10 \mathrm{mg} / \mathrm{kg} /$ day and modified based on liver function and concentration of TAC in serum. The dosage of MMF was initially determined per individual and ranged between 1.0 to $1.5 \mathrm{~g} / \mathrm{d}$. It was ceased if there were severe adverse effects and in long-time survivors who had a steady functioning graft 6 months after LT. At 6 months post-LT, the dosage of TAC was slowly and cautiously reduced, while carefully monitoring the allograft's function, to keep the TAC concentration as low as possible. For highrisk groups, such as HCC LT recipients who exceeded the Milan criteria, immunosuppressants were changed from oral TAC to oral sirolimus at our center, usually 1 month after surgery. Following the development of rejection, steroid pulse therapy was conducted. For hepatitis B (HB) virus-positive patients, 4,000 IU of HB immune globulin was given during the operation. Tenofovir was also given and HB immune globulin was frequently administered postoperatively to maintain an ideal antibody concentration against the $\mathrm{HB}$ surface antigen.

\section{Patient follow-up}

In the first 3 months of follow-up, the patients were followed-up weekly, then every 2 weeks for the following 3 months, and then monthly 6 months postoperatively. AFP level and an ultrasound of the liver were conducted during every follow-up. Computed tomography (CT) and liver enhancement magnetic resonance imaging (MRI) were performed every 3 months within 2 years after LT. Multidisciplinary teams provide regional therapies (surgery, radiofrequency ablation) or systematic treatment (targeted therapies, chemotherapy), and offer optimal supportive care for patients with HCC recurrence. The amount of time from surgery to the day of HCC recurrence or the final day of follow-up, including intrahepatic recurrence or distant metastasis, was determined as recurrence-free survival (RFS). Overall survival (OS) was determined as the period of time from the date of surgery until death or the final day of follow-up. The primary endpoint was RFS. During the study period, the median duration of followup was 13 months [with an interquartile range (IQR) of 621.3 months].

\section{Statistical analysis}

The continuous variables were compared with the MannWhitney $U$-test (for non-normal distributed data) or Student's $t$-test, and expressed as mean \pm criteria deviation. Categorical data are expressed by frequency and analyzed with 2-tailed $\chi^{2}$-test and Fisher's exact test. The KaplanMeier method was used to plot the survival curve, which was compared by the log-rank test. In the Cox proportional hazards model, possible risk factors, determined by univariate analysis $(\mathrm{P}<0.1)$, were added through a forward stepwise selection process, and risk factors were identified 


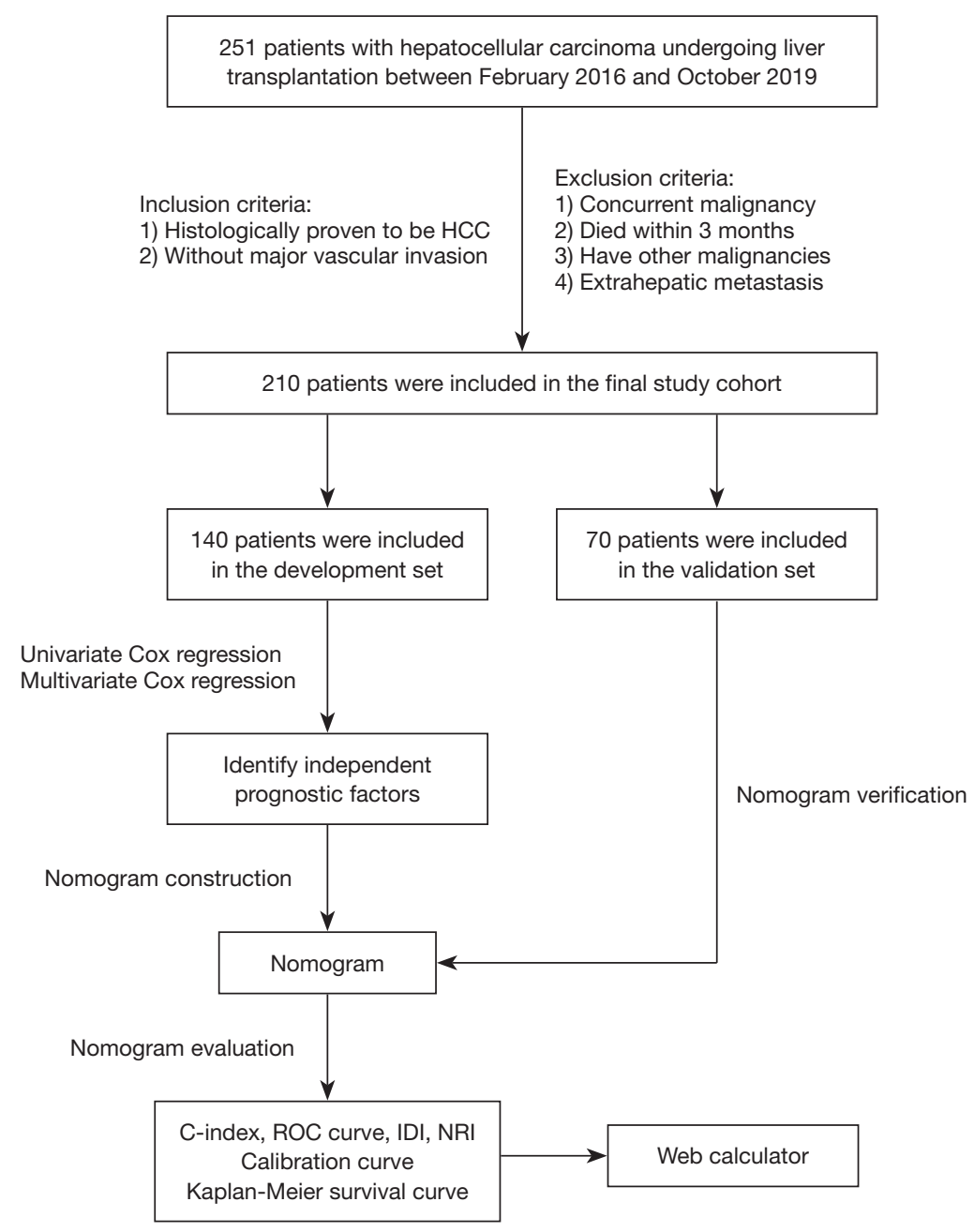

Figure 1 Flowchart of this study.

$(\mathrm{P}<0.05)$. Using these determined risk factors, we composed a nomogram to predict 1- and 2-year recurrence in HCC patients. To assess the nomogram's capacity to discriminate, we used the receiver-operating characteristic curve and concordance index (C-index), and evaluated the area under the curve (AUC). To compare the relationship between the estimated prospects and the factual outcomes, calibration curves were utilized. Calibration and discrimination were both assessed by bootstrapping with a 1,000 resamples. The accuracy of this new model was compared to the conventional American Joint Committee on Cancer (AJCC) staging system, and integrated discrimination improvement (IDI) and net reclassification improvement (NRI) were identified. R software (R Project for Statistical Computing, RRID:SCR_001905; version:3.3.0) was used to perform the statistical analyses. To identify the cutoff value for the continuous variables, a bioinformatics tool named X-tile (XTile,RRID:SCR_005602;version:3.6.1) was used (13).

\section{Results}

The flowchart of the study design is listed as follows (Figure 1).

\section{Demographics and pathological characteristics}

Data on patient sex and age were collected by reviewing the admission records. The number of tumors (single/ multiple), tumor envelope (yes/no), satellite lesions (yes/ no), vascular tumor thrombosis (yes/no), pathological grade (I/II/III), MVI classification (M0/M1/M2); medical history and AFP, ALT, aspartate aminotransferase (AST), $\gamma$-glutamyl transpeptidase (GGT), and international normalized ratio 
(INR) were collected from laboratory test reports, and the Model of End-Stage Liver Disease MELD (scores) were calculated. The median and range of the continuous variables are shown in Table 1. The $\chi^{2}$-test and independent samples $t$-test were carried out between the validation and the development sets; among the indicators of the two groups, no differences of statistical significance were found. To compare the cumulative recurrence rate among the two groups, a Kaplan-Meier curve was plotted, which showed no statistical difference. A summary of the baseline information is shown in Table 1. Figure 2 represents the Kaplan-Meier curve.

\section{Risk factor evaluation and nomogram construction}

The median duration of follow-up was 13 months (IQR, 6-21.3 months). Within this period of time, 62 patients (29.5\%) had recurrence of HCC. We first used X-tile software to determine the most suitable tangent point and to classify the continuous variables and then conducted multivariate and univariate regression analyses with the relevant content of the development set. In the univariate analysis, age, maximum tumor diameter, number of tumors, tumor thrombus, MVI, preoperative AFP, preoperative AST, preoperative GGT, and postoperative day 7 AFP, ALT, and INR $(\mathrm{P}<0.1)$ were added to the multivariate analysis. Age $(\mathrm{P}=0.004)$, maximum tumor diameter $(\mathrm{P}=0.015)$, thrombosis $(\mathrm{P}<0.001)$, MVI $(\mathrm{P}=0.033)$, and postoperative day $7 \operatorname{AFP}(\mathrm{P}=0.030)$ and ALT $(\mathrm{P}=0.048)$ remained significant in the multivariate analysis. The univariate and multivariate analyses are summarized in Table 2.

The nomogram predicting RFS was composed according to independent prognostic markers in the Cox model. The nomogram assigned the probability of RFS by accumulating the scores of every risk factor detected on the points scale. The total score visualized on the bottom scale specified the probability of 1- and 2-year RFS (Figure 3). Higher scores indicated worse prognosis.

In the development set, the C-index achieved 0.796, and the 1- and 2-year AUC achieved 0.799 and 0.801, respectively. In the validation set, the $\mathrm{C}$-index achieved 0.741, and the 1- and 2-year AUC achieved 0.732 and 0.705 , respectively (Figure 4).

The calibration curve demonstrated that the constructed nomogram model seemed to be well calibrated, and an adequate consensus existed among the observed and estimated probabilities of recurrences (Figure 5).

Evaluation of the accuracy demonstrated that the NRI of the 1- and 2-year follow-ups were 0.568 [95\% confidence interval (CI): 0.312-1.039] and 0.582 (95\% CI: 0.294-1.060), respectively, in the development set. Also in the development set, the IDI of the 1- and 2-year followups was $0.233(\mathrm{P}<0.001)$ and $0.271(\mathrm{P}<0.001)$, respectively. In the validation set, the NRI of the 1- and 2-year followups were 0.359 (95\% CI: -0.302 to 1.048 ) and 0.444 (95\% CI: -0.344 to 1.000$)$, respectively. Likewise, the IDI of the 1 - and 2-year follow-ups was $0.172(\mathrm{P}<0.001)$ and 0.209 $(\mathrm{P}<0.001)$, respectively, in the validation set. These findings suggest that our nomogram had more significant potential for the correct prediction of recurrence compared to the AJCC staging system.

The total score calculated by the nomogram was a continuous variable. Using $\mathrm{X}$-tile software, patients were divided into low- and high-risk groups. The predictive nomogram model that divided the groups into low and high risk showed not only significant differences in the cumulative recurrence rates but also significant differences in the cumulative survival rates, indicating that our model could also predict patient prognosis.

In the development set, the 1- and 2-year cumulative recurrence rates in the low-risk group were $19 \%$ and $31.5 \%$, and $75 \%$ and $93.7 \%$ in the high-risk group. The 1and 2-year cumulative survival rates in the low-risk group were $94.2 \%$ and $91.2 \%$, and $75 \%$ and $45 \%$ in the high-risk group.

In the validation set, the 1 - and 2-year cumulative recurrence rates in the low-risk group were $15.8 \%$ and $32.2 \%$, and $58.3 \%$ and $79.2 \%$ in the high-risk group. The $1-$ and 2-year cumulative survival rates in the low-risk group were $100 \%$ and $85.8 \%$, and $66.7 \%$ and $33.3 \%$ in the highrisk group (Figure 6).

For convenience, we constructed a web calculator: https://maensi.shinyapps.io/Huashan_surgery.

For instance, a 45 -year-old male (0 points) with a maximum tumor diameter of $7 \mathrm{~cm}$ (30.3 points), MVI of 1 (36.9 points), vascular tumor thrombus (78.8 points), AFP of $83 \mu \mathrm{g} / \mathrm{L}$ (6.2 points), and an ALT of $156 \mathrm{U} / \mathrm{L}$ (25.1 points) on postoperative day 7 would have a total score of 177.3 points. The estimated rate of recurrence is $18 \%$ in 1 year and $31 \%$ in 2 years (Figure 7). This calculated value could be used in the development of plans of treatment and in communication with the patient.

\section{Discussion}

The recurrence rate of HCC 5-year post-LT is $20-57.8 \%$, which is a crucial aspect that affects long-term survival. The 
Table 1 Baseline characteristics of the 210 transplantation patients

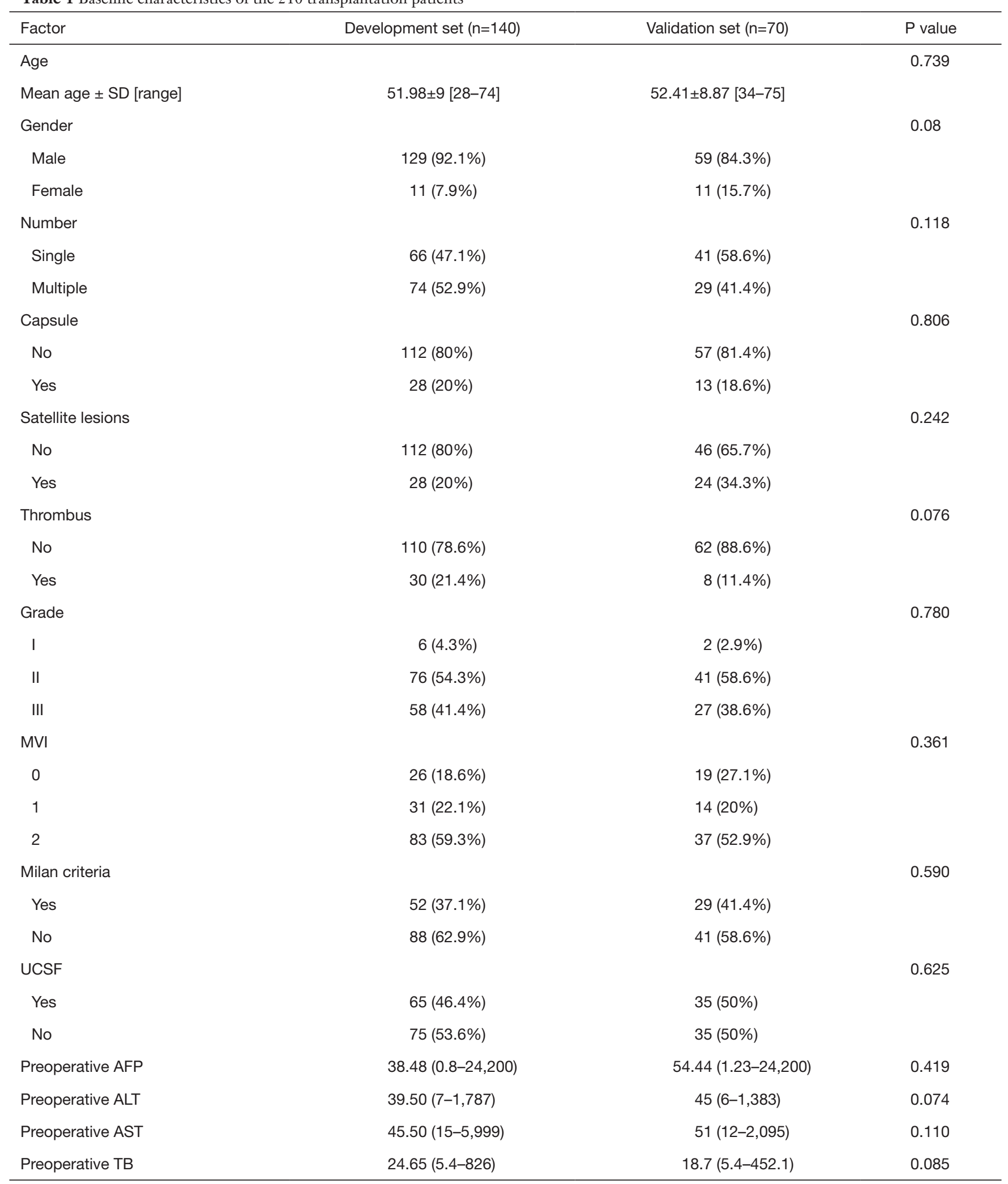

Table 1 (continued) 
Table 1 (continued)

\begin{tabular}{|c|c|c|c|}
\hline Factor & Development set $(n=140)$ & Validation set $(n=70)$ & $P$ value \\
\hline Preoperative INR & $1.195(0.92-2.81)$ & $1.12(0.89-2.81)$ & 0.128 \\
\hline Preoperative NLR & $2.537(0.519-53.765)$ & $3.115(0.623-44.714)$ & 0.107 \\
\hline Postoperative day 7 AFP & $8.96(0.25-24,200)$ & $11.55(0.05-24,200)$ & 0.447 \\
\hline Postoperative day 7 AST & $32.50(11-989)$ & $38(11-4,211)$ & 0.365 \\
\hline Postoperative day 7 TB & $45.70(9.8-522)$ & $39.85(12.8-302.5)$ & 0.331 \\
\hline Postoperative day 7 GGT & $106(27-913)$ & $112(33-790)$ & 0.901 \\
\hline Postoperative day 7 INR & $1.285(0.98-4.47)$ & $1.245(1.03-2.52)$ & 0.166 \\
\hline
\end{tabular}

AFP, alpha-fetoprotein; ALT, alanine aminotransferase; AST, aspartate aminotransferase; Cl, confidence interval; GGT, $\gamma$-glutamyl transpeptidase; HR, hazard ratio, INR, international normalized ratio; MELD, Model of End-Stage Liver Disease; MVI, microvascular invasion; NLR, neutrophil lymphocyte ratio; SD, standard deviation; TB, total bilirubin; UCSF, University Of California ,San Francisco.

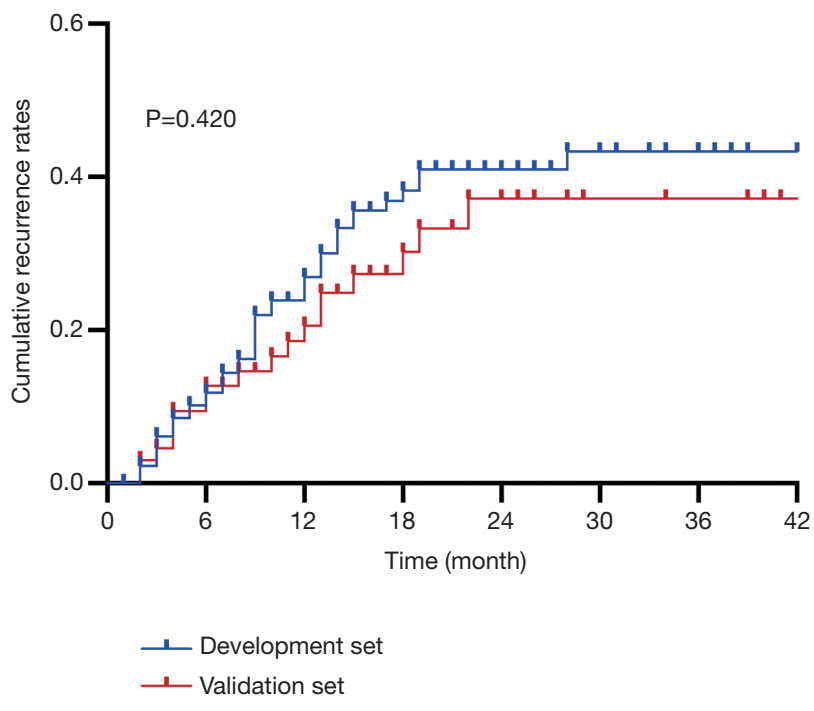

Figure 2 Differences in cumulative recurrence rates between the development set and the validation set.

ever-expanding criteria for LT can benefit more patients, but can also increase the risk of recurrence post-LT $(4,7)$. However, patients who met the strict Milan criteria still had a nearly $20 \%$ chance of relapse or metastasis after surgery. A variety of different clinical pathology parameters, including patient factors, tumor factors, and perinatal treatment factors, have been reported to be associated with post-LT relapse and metastasis. However, the predictive effectiveness of individual factors is limited. The scoring system derived from a collection of factors is a better choice.

Researchers at the University of California used data derived from 721 patients who met the Milan criteria to propose a prognostic scoring system (10). The following three variables were found to be related to disease recurrence: MVI, AFP level in serum at LT, and maximum nodule diameter as well as the sum of nodules. These three variables formed the basis of a scoring system that could predict the probability of recurrence. However, the study participants were early HCC patients who fulfilled the Milan criteria, but could not meet the ever-expanding needs of the current criteria for HCC LT. Moreover, the evaluation and predictive index were non-linear, and therefore could not determine individual prediction.

The nomogram has the advantage of high accuracy, can consider various clinical pathology factors, and produce individualized risk predictions. Several researchers have applied nomograms to predict HCC recurrence and metastasis after LT. However, they either only included a Western population that mainly suffered from a history of liver disease as a result of HCV infection or did not include patients' postoperative liver function during the perioperative period.

Using multi-factorial regression analysis, we found that postoperative 7 days AFP and ALT, age, maximum tumor diameter, MVI, and portal venous tumor thrombus were independent risk factors associated with RFS. 
Page 8 of 15

Ma et al. A nomogram with recurrence prediction in HCC patients

Table 2 Evaluation of variables related to recurrence-free survival

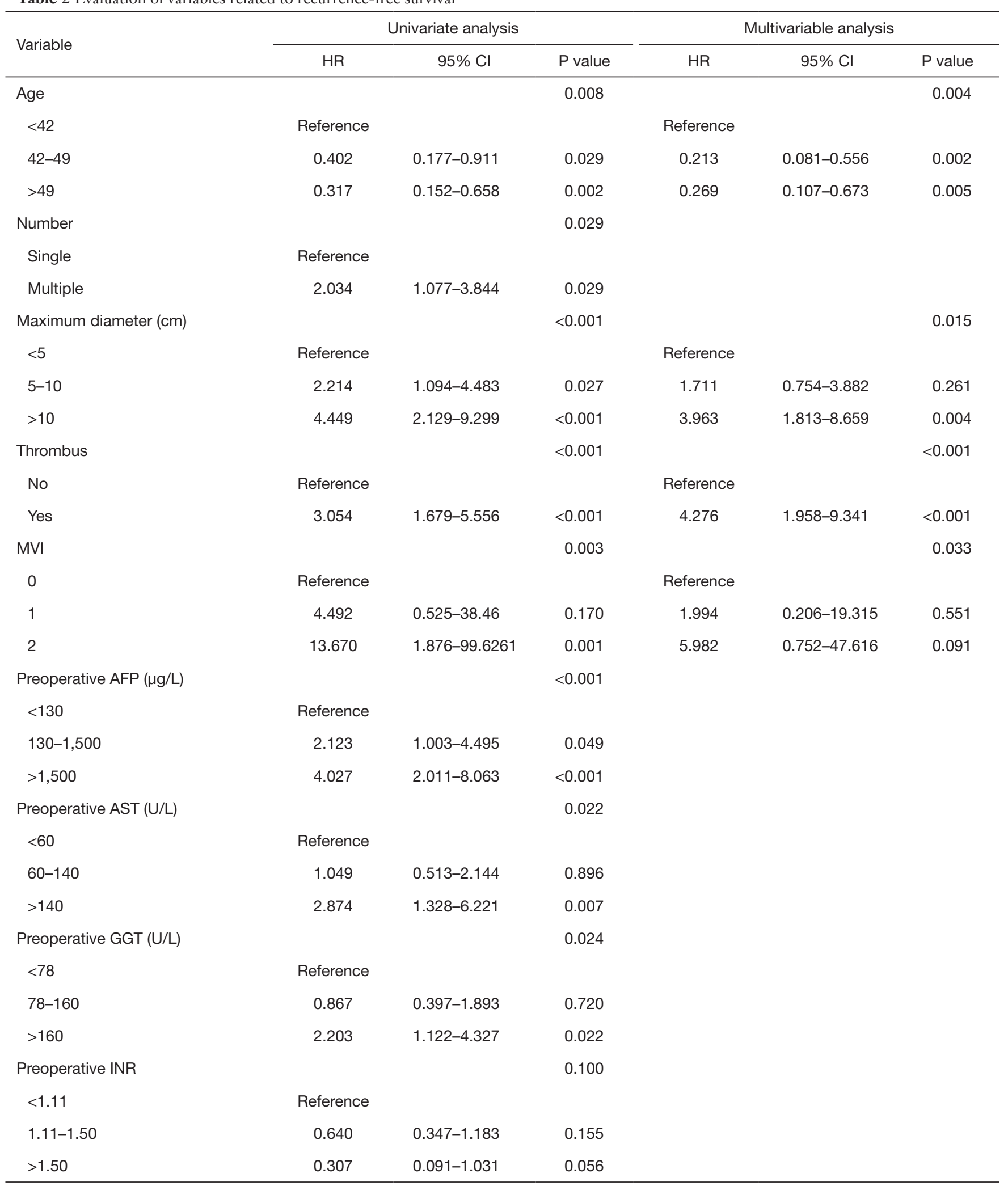

Table 2 (continued) 
Table 2 (continued)

\begin{tabular}{|c|c|c|c|c|c|c|}
\hline Variable & \multicolumn{3}{|c|}{ Univariate analysis } & \multicolumn{3}{|c|}{ Multivariable analysis } \\
\hline Postoperative day 7 AFP ( $\mu \mathrm{g} / \mathrm{L})$ & & & $<0.001$ & & & 0.030 \\
\hline$<55$ & Reference & & & Reference & & \\
\hline $55-835$ & 2.516 & $1.277-4.955$ & 0.008 & 1.117 & $0.455-2.742$ & 0.810 \\
\hline Postoperative day 7 ALT (U/L) & & & 0.055 & & & 0.048 \\
\hline$<83$ & Reference & & & Reference & & \\
\hline 83-280 & 1.453 & $0.703-3.001$ & 0.313 & 1.545 & $0.723-3.300$ & 0.261 \\
\hline$>280$ & 2.747 & $1.189-6.345$ & 0.018 & 2.990 & $1.233-7.246$ & 0.015 \\
\hline $1.36-1.67$ & 0.395 & $0.153-1.020$ & 0.055 & & & \\
\hline$>1.67$ & 2.257 & $1.065-4.781$ & 0.034 & & & \\
\hline
\end{tabular}

AFP, alpha-fetoprotein; ALT, alanine aminotransferase; AST, aspartate aminotransferase; CI, confidence interval; GGT, $\gamma$-glutamyl transpeptidase; HR, hazard ratio, INR, international normalized ratio; MVI, microvascular invasion.

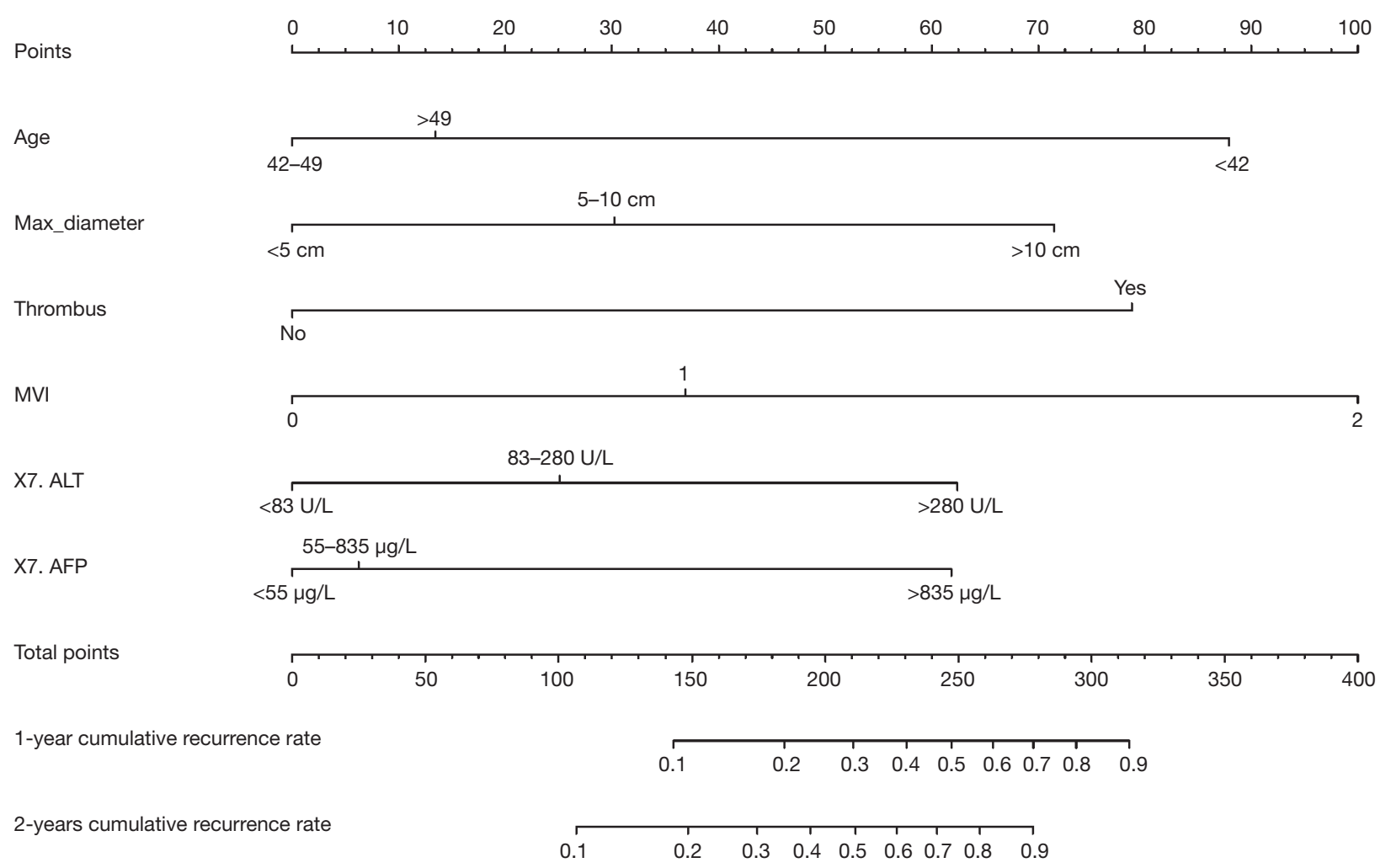

Figure 3 Prognostic nomogram for the prediction of recurrence-free survival for hepatocellular carcinoma post-transplantation. For every predictor, a straight vertical line upward was projected to identify the points. Total points bar was used to plot the accumulated points, and a straight vertical line yielded the 1- and 2-year predicted post-transplantation survival risk. 
A

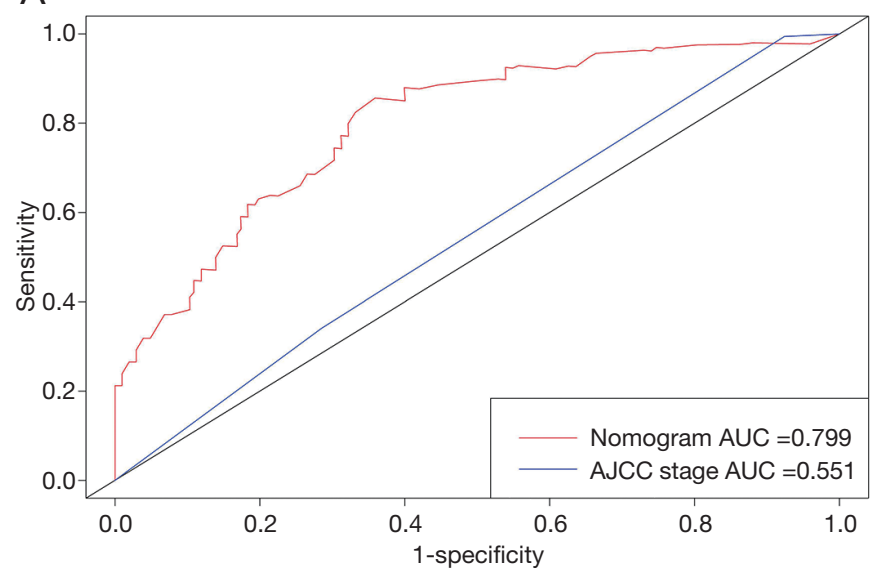

C

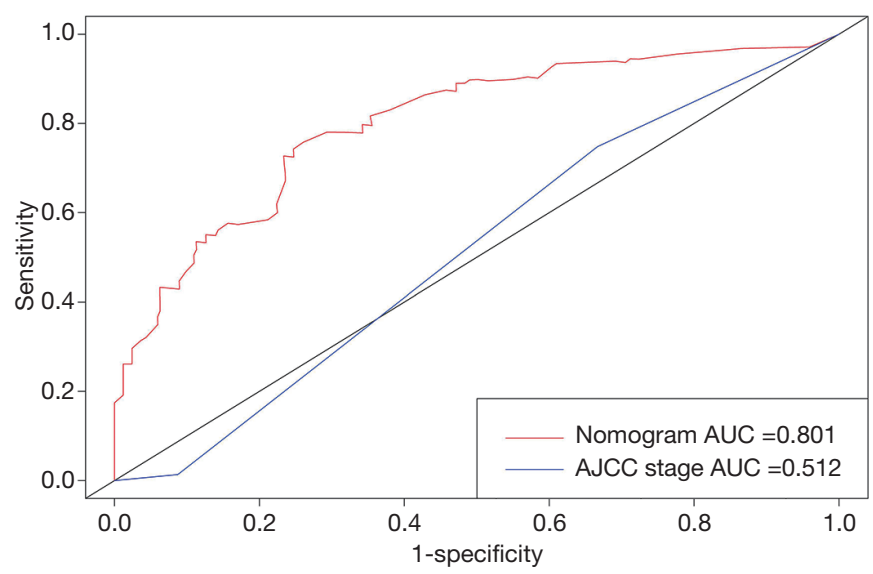

Ma et al. A nomogram with recurrence prediction in HCC patients

B

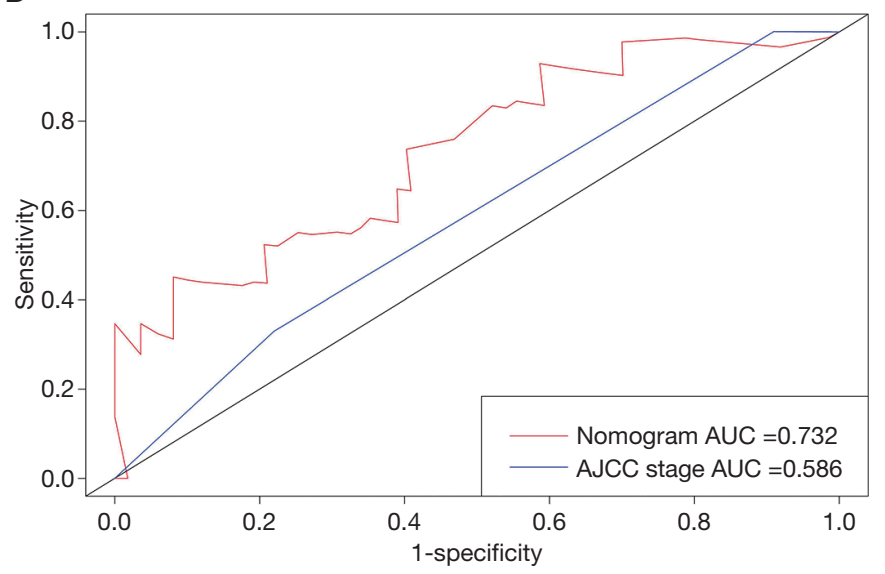

D

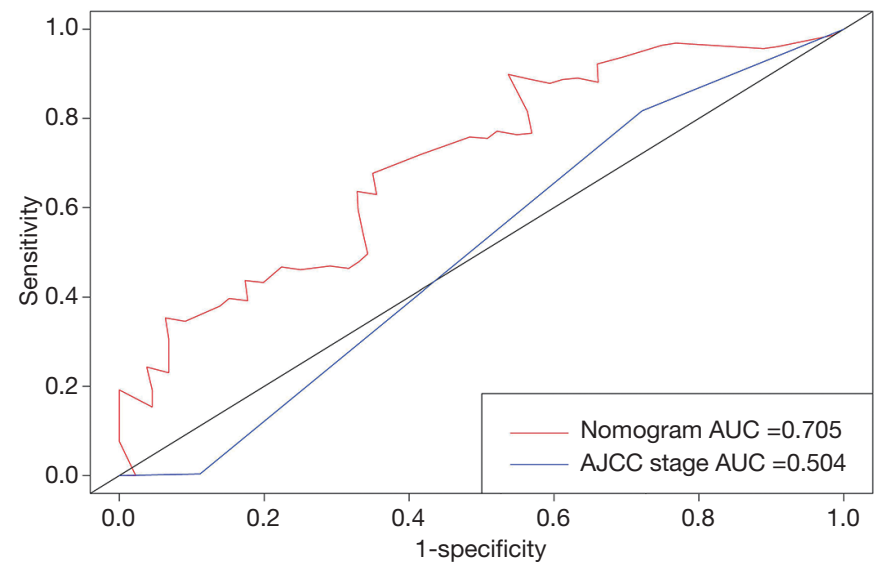

Figure 4 Prognostic distinction and estimative performance of the nomogram. (A) 1-year recurrence-free survival (RFS) in the development set; (B) 1-year RFS in the validation set; (C) 2-year RFS in the development set; (D) 2-year RFS in the validation set.

Although many biomarkers have been studied over the past few decades, serological testing for HCC recurrence still mostly depends on the traditional tumor marker AFP $(14,15)$. Moreover, the serum AFP level is a crucial factor affecting HCC cell invasion, metastasis, and prognosis $(16,17)$. Other studies, such as those of Bilbao et al. and Krasnodębski et al., found that elderly patients with HCC had shorter OS and RFS after LT. Older age was considered a critical risk factor for recurrence of tumor in patients with HCC $(18,19)$. Vascular aggression is also considered a critical step in the recurrence of HCC, including macrovascular aggression visibility and MVI. MVI, which is confirmed by preoperative imaging, can be accurately diagnosed. Vascular aggression exists in approximately $35-50 \%$ of patients (20), and is diagnosed with the trunk in $15-30 \%$ of cases (21). Once diagnosed, the opportunity for radical removal and transplantation is lost, and the prognosis is poor. Vascular aggression is currently regarded as a vital adverse prognostic factor because it increases the possibility of cancer cells being released into the vascular system, resulting in a higher probability of recurrence and metastasis. In this situation, LT might only be beneficial in cases where there is an excellent biological response in preoperative treatment or in cases where liver function is decompensated $(20,22,23)$.

As an essential link to invasive metastasis, MVI has a high incidence of $15-57.1 \%$, and has become a recognized independent prognostic risk factor for HCC (24). Studies have reported that it can serve as a value of prediction for HCC patients who received hepatectomy or LT (25). In a previously published study, 902 HCC LT patients were found to have an MVI-positive rate of $22.1 \%$, and MVI was a critical indicator affecting the prognosis of HCC LT patients (26). 
A

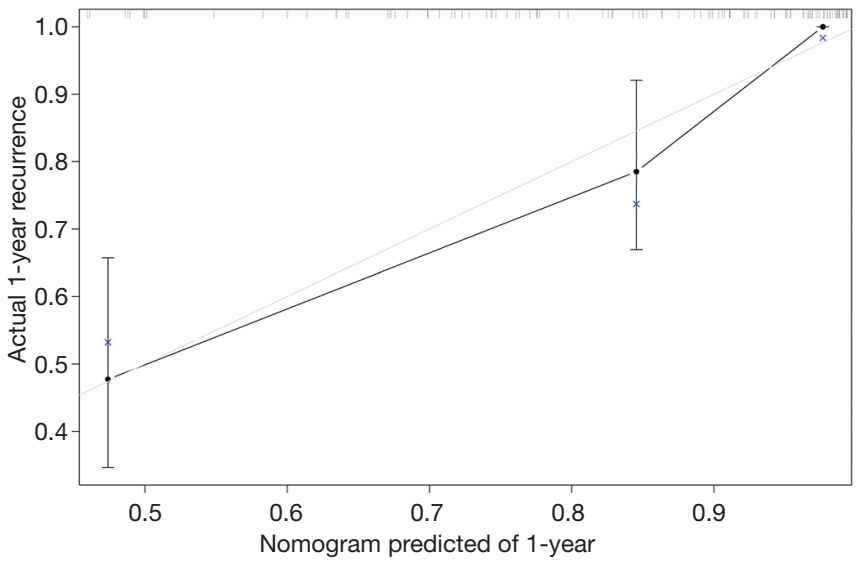

C

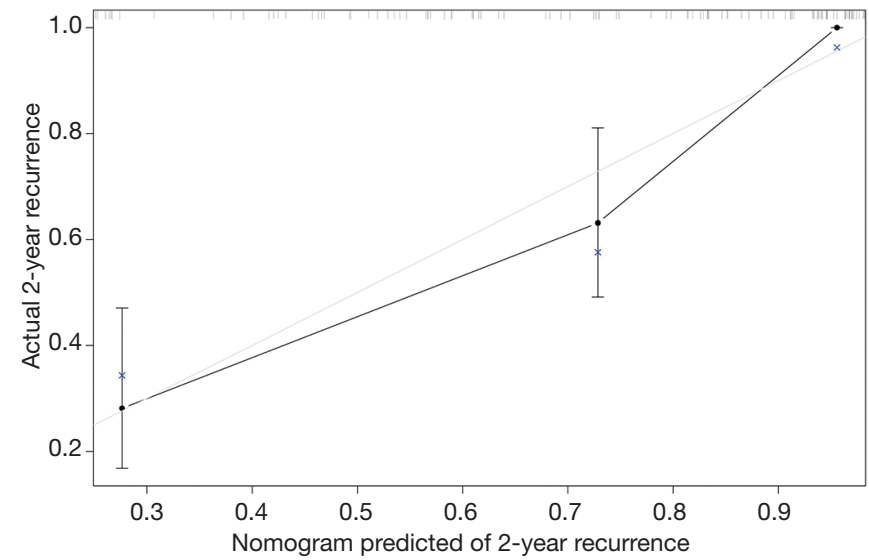

B

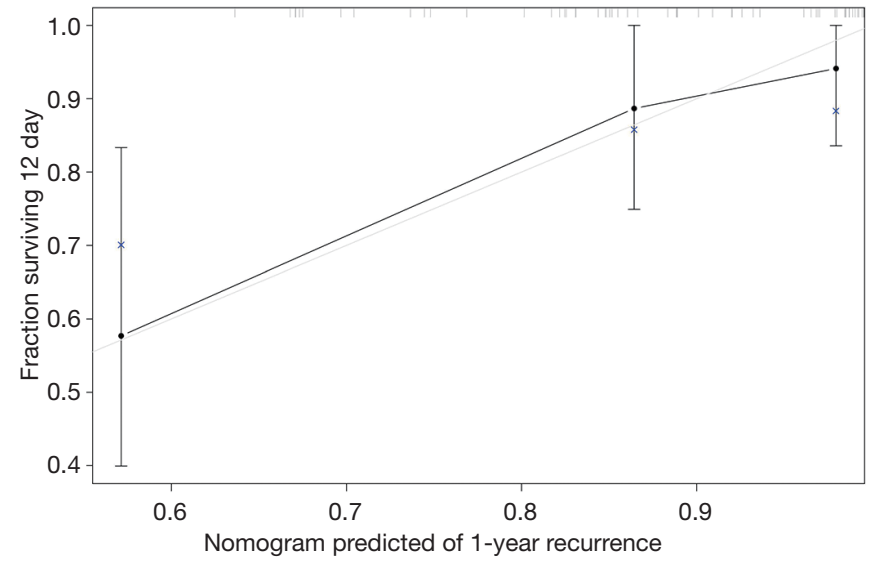

D

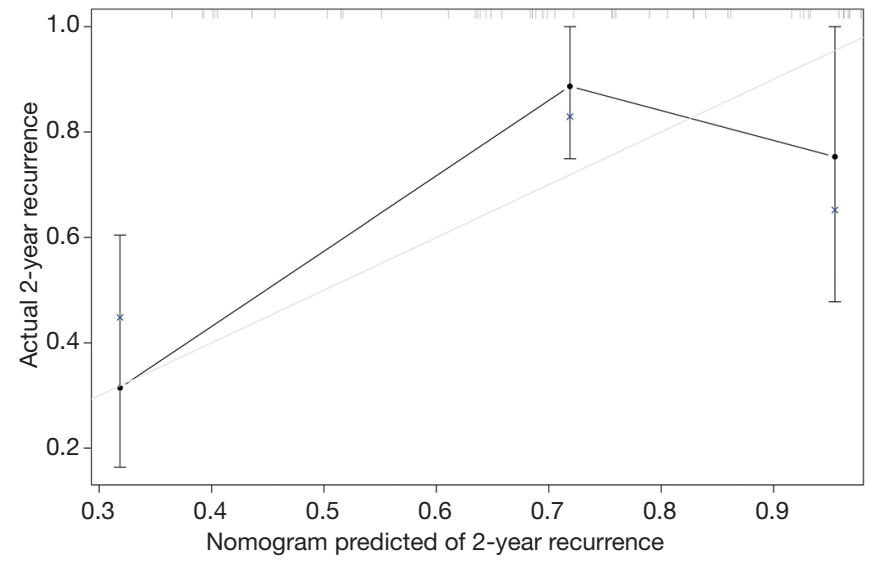

Figure 5 Calibration of 1- and 2-year recurrence-free survival (RFS) in the development and validation sets. (A) 1-year RFS in the development set; (B) 1-year RFS in the validation set; (C) 2-year RFS in the development set; (D) 2-year RFS in the validation set.

The liver function index is also associated with HCC, as abnormalities in liver function that persist after surgery may lead to inflammation, immune microenvironment disorder, and oxidative stress. The postoperative delayed recovery of liver function can lead to an increased risk of recurrence of HCC $(27,28)$. ALT has been widely used to assess chronic hepatitis activity and has been found to be closely related to the prognosis of HCC (29-32).

The tumor thrombus is a distinct predictor of mortality related to early recurrence, and also a significant adverse factor of prognosis (33).

The present study included patients met and did not meet the LT criteria. The predictive nomogram model was based on multivariate analysis. This nomogram chart accurately estimated the risk of recurrence post-LT and can be used to determine enhanced surveillance and postoperative treatment of patients who have a high risk of recurrence. The model combines serum biomarkers (AFP, ALT) and pathological information (MVI, cancer thrombus), which significantly improve the potential to estimate recurrence after LT compared with individually determined imaging criteria. We produced a web calculator that could efficiently predict recurrence post-LT and serve as a guide to the frequency of post-LT monitoring and help the surgeon determine individual treatment strategies for patients with a high risk of relapse post-LT.

\section{Limitations}

The nomogram is expected to be a valuable tool for detecting patients at high risk of relapse and metastasis after HCC LT. However, the present study has some limitations. 
A

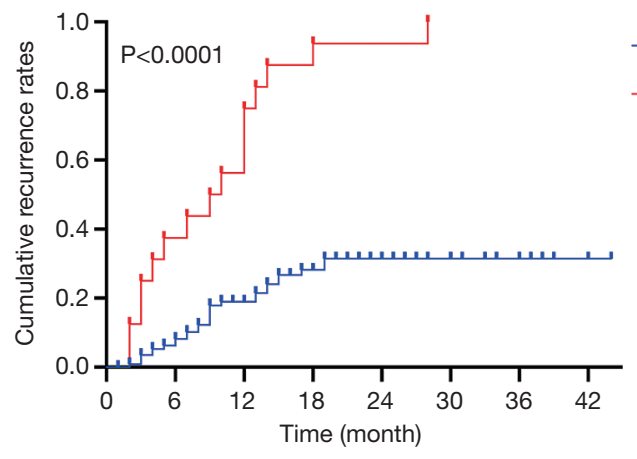

$\begin{array}{lllllllll}\text { Low risk: } & 124 & 92 & 66 & 44 & 31 & 20 & 9 & 1\end{array}$

High risk: $\begin{array}{lllllllll}16 & 10 & 4 & 1 & 1 & 0 & 0 & 0\end{array}$

C

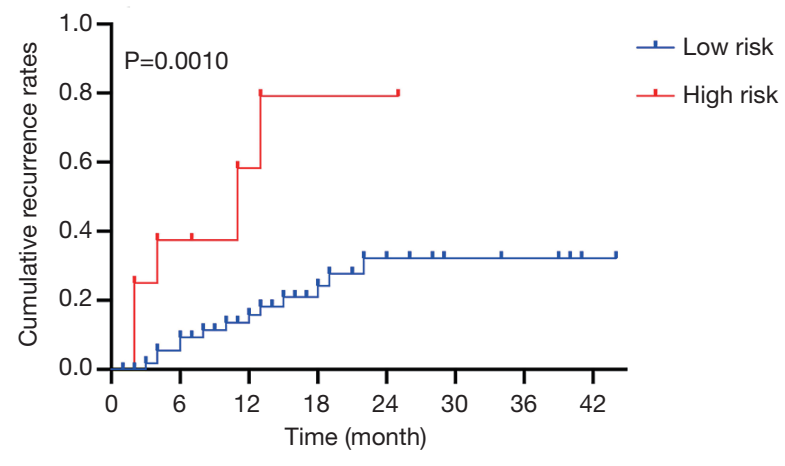

$\begin{array}{lllllllll}\text { Low risk: } & 62 & 47 & 35 & 22 & 12 & 8 & 5 & 1\end{array}$

$\begin{array}{lllllllll}\text { High risk: } & 8 & 5 & 2 & 1 & 1 & 0 & 0 & 0\end{array}$

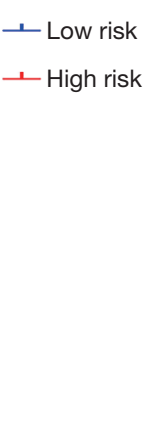

B

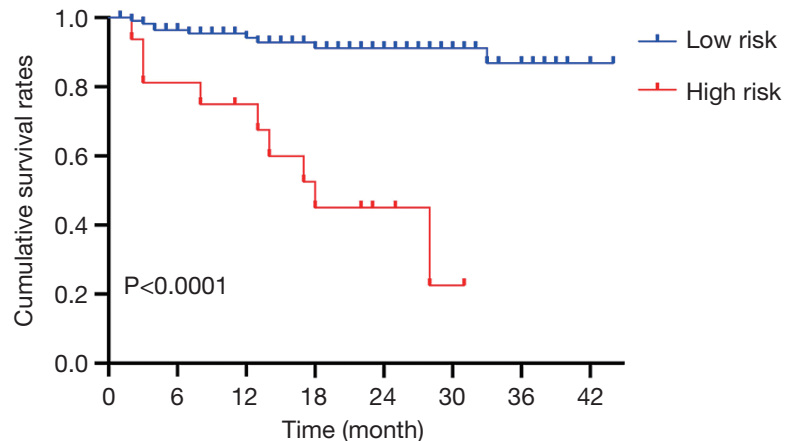

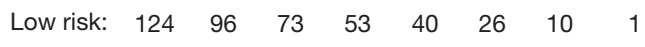

High risk: $\begin{array}{llllllll}16 & 13 & 10 & 5 & 3 & 1 & 0 & 0\end{array}$

D

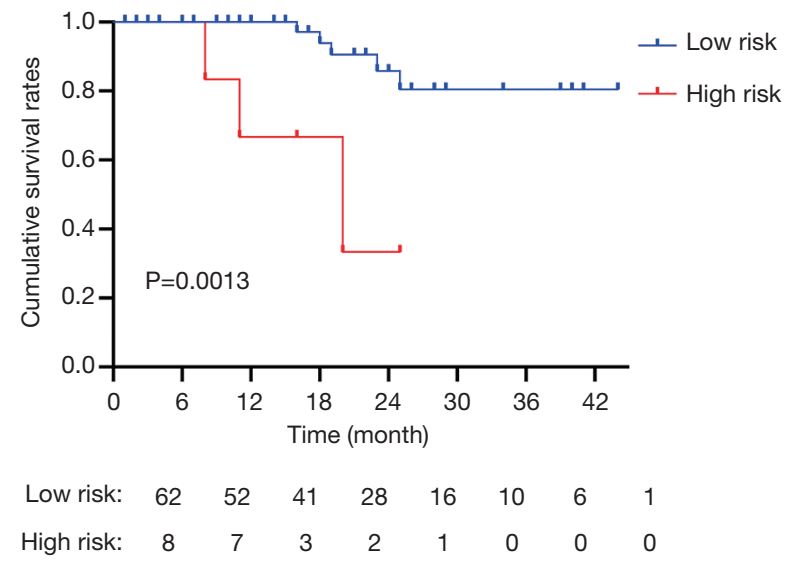

Figure 6 Comparison of cumulative survival and recurrence rates in the validation and development sets. (A) Cumulative recurrence rate in the development set; (B) Cumulative survival rate in the development set; (C) Cumulative recurrence rate in the validation set; (D) Cumulative survival rate in the validation set.

The present study was a retrospective study with limited samples and required external verification. While predictive models are not able to replace the evidence collected from prospective randomized clinical trials, they can be useful aids in clinical decisions-making, where the availability of clinical trial data and the best treatment management remain controversial.

\section{Conclusions}

The nomogram, which was based on clinical-pathological factors, showed good accuracy in the prediction of prognostic recurrence, and can guide individual follow-up and treatment.

\section{Acknowledgments}

The abstract of this paper was presented at the 28th International Congress of the Transplantation Society as a poster presentation. Web link: https://virtual.tts.org/virtual/ lecture/1057

Funding: This study was supported by grants from the National Science and Technology Major Projects (No. 2017ZX10203205-002-004 and 2017ZX10203205-003003), the National Natural Science Foundation of China (No. 81873874 and 81773089) and Clinical Research Plan of SHDC (No. SHDC2020CR2021B). These funds provided support to researchers for data collection, patient follow-up, and data analysis. 
A

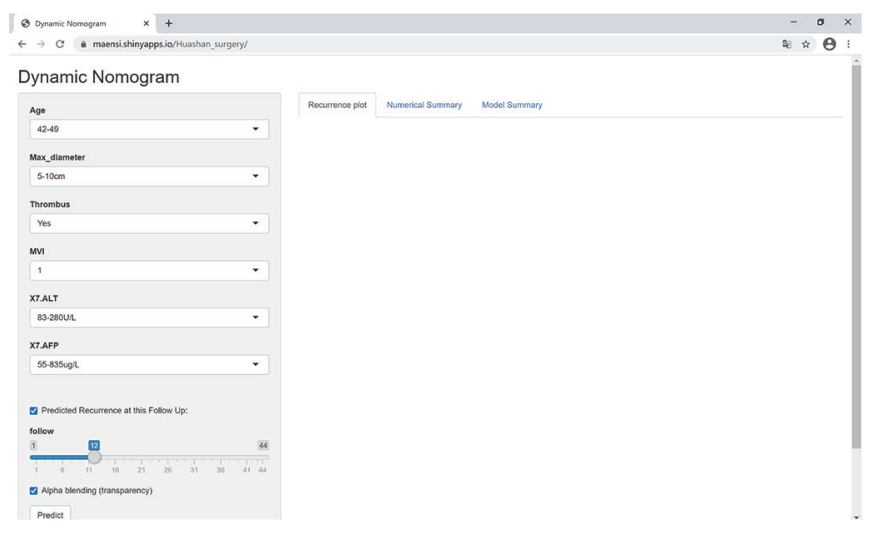

C

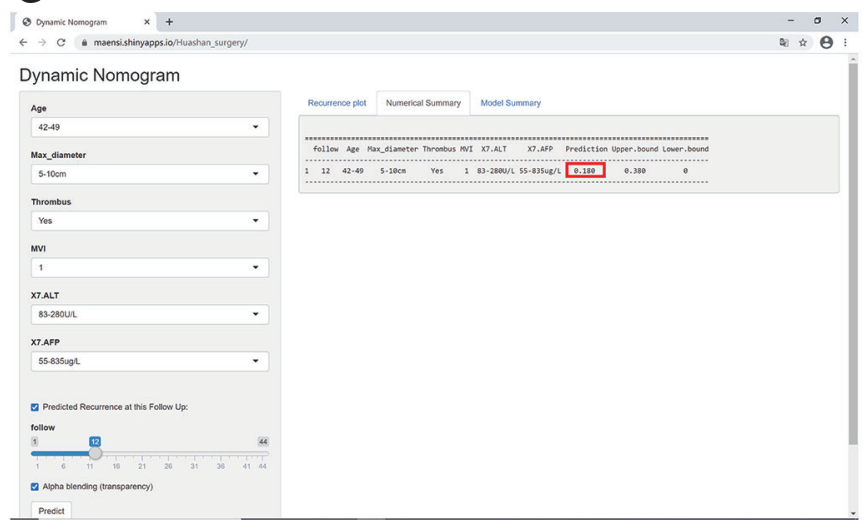

Figure 7 Web calculator case presentation.

\section{Footnote}

Reporting Checklist: The authors have completed the TRIPOD reporting checklist. Available at http://dx.doi. org/10.21037/atm-21-334

Data Sharing Statement: Available at http://dx.doi. org/10.21037/atm-21-334

Conflicts of Interest: All authors have completed the ICMJE uniform disclosure form (available at http://dx.doi. org/10.21037/atm-21-334). The authors have no conflicts of interest to declare.

Ethical Statement: The authors are accountable for all aspects of the work in ensuring that questions related to the accuracy or integrity of any part of the work are appropriately investigated and resolved. The present, retrospective study was performed according to the Declaration of Helsinki (as revised in 2013). This study was
B

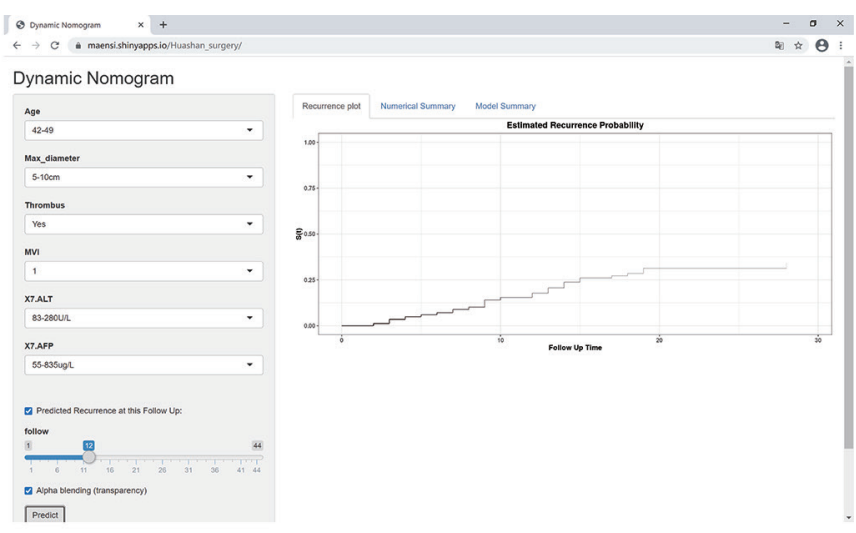

D

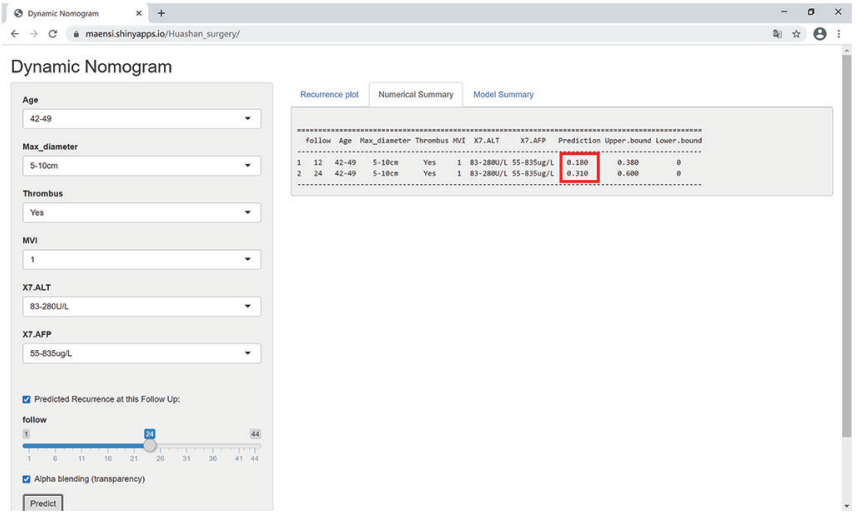

approved by the Ethics Committee of Huashan Hospital Affiliated to Fudan University (No. KY-2019-511). Written consent to publish this information has been obtained from all participants.

Open Access Statement: This is an Open Access article distributed in accordance with the Creative Commons Attribution-NonCommercial-NoDerivs 4.0 International License (CC BY-NC-ND 4.0), which permits the noncommercial replication and distribution of the article with the strict proviso that no changes or edits are made and the original work is properly cited (including links to both the formal publication through the relevant DOI and the license). See: https://creativecommons.org/licenses/by-nc-nd/4.0/.

\section{References}

1. Bray F, Ferlay J, Soerjomataram I, et al. Global cancer statistics 2018: GLOBOCAN estimates of incidence and 
mortality worldwide for 36 cancers in 185 countries. CA Cancer J Clin 2018;68:394-424.

2. Yang JD, Hainaut P, Gores GJ, et al. A global view of hepatocellular carcinoma: trends, risk, prevention and management Nat Rev Gastroenterol Hepatol 2019;16:589-604.

3. Mazzaferro V, Regalia E, Doci R, et al. Liver transplantation for the treatment of small hepatocellular carcinomas in patients with cirrhosis. N Engl J Med 1996;334:693-9.

4. Xu X, Lu D, Ling Q, et al. Liver transplantation for hepatocellular carcinoma beyond the Milan criteria. Gut 2016;65:1035-41.

5. Yao FY, Ferrell L, Bass NM, et al. Liver transplantation for hepatocellular carcinoma: expansion of the tumor size limits does not adversely impact survival. Hepatology 2001;33:1394-403.

6. Sapisochin G, Bruix J. Liver transplantation for hepatocellular carcinoma: Outcomes and novel surgical approaches. Nat Rev Gastroenterol Hepatol 2017;14:203-17.

7. Zavaglia C, De Carlis L, Alberti AB, et al. Predictors of long-term survival after liver transplantation for hepatocellular carcinoma. Am J Gastroenterol 2005;100:2708-16.

8. Verna EC, Patel YA, Aggarwal A, et al. Liver transplantation for hepatocellular carcinoma: Management after the transplant. Am J Transplant 2020;20:333-47.

9. Mehta N, Heimbach J, Harnois DM, et al. Validation of a Risk Estimation of Tumor Recurrence After Transplant (RETREAT) score for hepatocellular carcinoma recurrence after liver transplant. JAMA Oncol 2017;3:493-500.

10. Agopian VG, Harlander-Locke M, Zarrinpar A, et al. A novel prognostic Nomogram accurately predicts hepatocellular carcinoma recurrence after liver transplantation: analysis of 865 consecutive liver transplant recipients. J Am Coll Surg 2015;220:416-27.

11. Shen J, Li C, Wen J, et al. Nomograms to predict prognosis of hepatocelluar carcinoma without major vascular invasion after primary liver transplantation. Oncotarget 2014;5. Available online: https://www. oncotarget.com/article/24042/

12. Huang LF, Wan P, Xu DW, et al. Nomogram predicting pulmonary metastasis of hepatocellular carcinoma after liver transplantation. Oncotarget 2017;9:2425-34.

13. Camp RL, Dolled-Filhart M, Rimm DL. X-tile: a new bio-informatics tool for biomarker assessment and outcome-based cut-point optimization. Clin Cancer Res
2004;10:7252-9.

14. Pavlides S, Tsirigos A, Migneco G, et al. The autophagic tumor stroma model of cancer: Role of oxidative stress and ketone production in fueling tumor cell metabolism. Cell Cycle 2010;9:3485-505.

15. Koteish A, Thuluvath PJ. Screening for hepatocellular carcinoma. J Vasc Interv Radiol 2002;13:S185-S190.

16. Lu Y, Zhu M, Li W, et al. Alpha fetoprotein plays a critical role in promoting metastasis of hepatocellular carcinoma cells. J Cell Mol Med 2016;20:549-58.

17. Duvoux C, Roudot-Thoraval F, Decaens T, et al. Liver transplantation for hepatocellular carcinoma: a model including $\alpha$-fetoprotein improves the performance of Milan criteria. Gastroenterology 2012;143:986-94.e3; quiz e14-5.

18. Krasnodębski M, Grąt M, Masior $€$, et al. Differential impact of risk factors for tumor recurrence in hepatitis $\mathrm{B}$ and hepatitis $\mathrm{C}$ virus-infected patients undergoing liver transplantation for hepatocellular carcinoma. Ann Transplant 2015;20:70-5.

19. Bilbao I, Dopazo C, Lazaro JL, et al. Our experience in liver transplantation in patients over $65 \mathrm{yr}$ of age. Clin Transplant 2008;22:82-8.

20. Cerrito L, Annicchiarico BE, Iezzi R, et al. Treatment of hepatocellular carcinoma in patients with portal vein tumor thrombosis: Beyond the known frontiers. World J Gastroenterol 2019;25:4360-82.

21. Minagawa M, Makuuchi M, Takayama T, et al. Selection criteria for hepatectomy in patients with hepatocellular carcinoma and portal vein tumor thrombus. Ann Surg 2001;233:379-84.

22. von Felden J, Villanueva A. Role of Molecular Biomarkers in Liver Transplantation for Hepatocellular Carcinoma. Liver Transpl 2020;26:823-31.

23. Liu PH, Huo TI, Miksad RA. Hepatocellular Carcinoma with Portal Vein Tumor Involvement: Best Management Strategies. Semin Liver Dis 2018;38:242-51.

24. Rodríguez-Perálvarez M, Luong TV, Andreana L, et al. A systematic review of microvascular invasion in hepatocellular carcinoma: diagnostic and prognostic variability. Ann Surg Oncol 2013;20:325-39.

25. Iguchi T, Shirabe K, Aishima S, et al. New pathologic stratification of microvascular invasion in hepatocellular carcinoma: predicting prognosis after living-donor liver transplantation. Transplantation 2015;99:1236-42.

26. Onaca N, Davis GL, Jennings LW, et al. Improved results of transplantation for hepatocellular carcinoma: a report from the International Registry of Hepatic Tumors in 
Liver Transplantation. Liver Transpl 2009;15:574-80.

27. Zendejas-Ruiz I, Hemming AW, Chen C, et al. Recurrent hepatocellular carcinoma in liver transplant recipients with hepatitis C. J Gastrointest Cancer 2012;43:229-35.

28. Ju MJ, Qiu SJ, Fan J, et al. Preoperative serum gammaglutamyl transferase to alanine aminotransferase ratio is a convenient prognostic marker for Child-Pugh A hepatocellular carcinoma after operation. J Gastroenterol 2009;44:635-42.

29. Kim HC, Nam CM, Jee SH, et al. Normal serum aminotransferase concentration and risk of mortality from liver diseases: prospective cohort study. BMJ 2004;328:983.

30. Cheung YS, Chan HLY, Wong J, et al. Elevated perioperative transaminase level predicts intrahepatic recurrence in hepatitis B-related hepatocellular carcinoma

Cite this article as: Ma E, Li J, Xing H, Li R, Shen C, Zhang Q, Ma Z, Tao Y, Qin L, Zhao J, Wang Z. Development of a predictive nomogram for early recurrence of hepatocellular carcinoma in patients undergoing liver transplantation. Ann Transl Med 2021;9(6):468. doi: 10.21037/atm-21-334 after curative hepatectomy. Asian J Surg 2008;31:41-9.

31. Tarao K, Rino Y, Takemiya S, et al. Serum alanine aminotransferase levels and survival after hepatectomy in patients with hepatocellular carcinoma and hepatitis $\mathrm{C}$ virus-associated liver cirrhosis. Cancer Sci 2003;94:1083-90.

32. Tarao K, Rino Y, Takemiya S, et al. Close association between high serum ALT and more rapid recurrence of hepatocellular carcinoma in hepatectomized patients with $\mathrm{HCV}$-associated liver cirrhosis and hepatocellular carcinoma. Intervirology 2000;43:20-6.

33. Lim C, Compagnon P, Sebagh M, et al. Hepatectomy for hepatocellular carcinoma larger than $10 \mathrm{~cm}$ : preoperative risk stratification to prevent futile surgery. HPB (Oxford) 2015;17:611-23.

(English Language Editor: R. Scott) 